\title{
Il progetto Cities Changing Diabetes
}

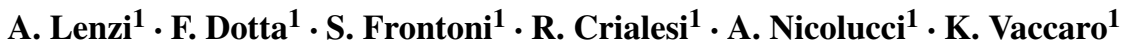

C Springer International Publishing AG 2017

\begin{abstract}
Sommario La popolazione urbana è in crescita costante: ogni anno aumenta di circa 60 milioni di persone, soprattutto nei Paesi a medio reddito. Proiezioni di popolazione mostrano che nei prossimi 30 anni la crescita globale avverrà solo nelle aree urbane. I cambiamenti demografici in corso (urbanizzazione, peggioramento degli stili di vita, invecchiamento della popolazione e isolamento sociale) si riflettono in una crescita costante della prevalenza di diabete. Il diabete è una delle patologie croniche a più ampia diffusione nel mondo e costituisce una delle più rilevanti e costose malattie sociali della nostra epoca. Nelle grandi città vivono oggi due terzi delle persone affette da diabete; da qui la necessità di mettere in atto nelle aree urbane una strategia integrata, finalizzata a costruire un'idea di città come "promotore della salute". L'obiettivo del progetto è creare un movimento unitario in grado di stimolare i decisori politici a considerare come prioritario il tema dell'urban diabetes. Il programma Cities Changing Diabetes ${ }^{\circledR}$ sottolinea la necessità di agire in considerazione del crescente numero di persone con diabete e del conseguente onere economico e sociale che tutto ciò comporta. Roma è stata inserita nel programma nel 2017, diventando per i prossimi anni oggetto di studi su urbanizzazione e diabete Tipo 2 e nello stesso tempo Città simbolo mondiale nella lotta a questa importante patologia.
\end{abstract}

Parole chiave Diabete mellito $\cdot$ Promozione della salute Urban Health

A. Lenzi

andrea.lenzi@uniroma1.it; url: http://healthcitythinktank.org

1 Health City Institute, Roma, Italia

\section{Introduzione}

Cento anni fa solo due persone su dieci nella popolazione mondiale vivevano nelle aree urbane. Nella metà del $21^{\circ}$ secolo questo numero arriverà a sette. La popolazione urbana, pertanto, è in crescita costante: ogni anno aumenta di circa 60 milioni di persone, soprattutto nei Paesi a medio reddito. Proiezioni di popolazione mostrano che nei prossimi 30 anni la crescita globale avverrà virtualmente soltanto nelle aree urbane. Tuttavia, secondo quanto riportato dall'UNICEF, circa un terzo della popolazione urbana mondiale vive nei bassifondi, dove si concentrano povertà, emarginazione e discriminazione; entro il 2020 le persone che vivranno in insediamenti non ufficiali e negli slum saranno quasi 1,4 miliardi. Quasi il 10\% della popolazione urbana, inoltre, vive in megalopoli, città con oltre 10 milioni di abitanti che si sono moltiplicate in tutto il pianeta, ma la quota maggiore dell'incremento in ambiente urbano si sta verificando non nelle megalopoli ma in città più piccole. Il notevole incremento della popolazione nelle aree urbane è legato anche ai fenomeni migratori; le regioni urbane dell'Unione europea, fatta eccezione per la Francia, tendono a registrare, infatti, gli incrementi demografici più elevati a causa del saldo migratorio.

La migrazione della popolazione verso le aree urbane si accompagna a cambiamenti sostanziali degli stili di vita rispetto al passato; cambiano le abitudini, cambia il modo di vivere, i lavori sono sempre più sedentari, l'attività fisica diminuisce. Fattori sociali e culturali che rappresentano un potente volano per obesità e diabete. Pertanto, sebbene l'epidemia della cronicità sia universalmente considerata come una delle più importanti sfide per i sistemi sanitari e affondi le sue origini nella diffusione di pochi fattori di rischio legati a stili di vita non salutari, ancora tanto c'è da fare per diffondere una cultura della prevenzione che miri a sviluppare consapevolezza nelle scelte di salute delle persone. 


\section{Dall'Urban Health all'Urban Diabetes}

I cambiamenti demografici in corso, che includono l'urbanizzazione, il peggioramento degli stili di vita, l'invecchiamento della popolazione e l'isolamento sociale si riflettono in una crescita costante della prevalenza di diabete. Il diabete è una delle patologie croniche a più ampia diffusione nel mondo, in particolare nei Paesi industrializzati, e costituisce una delle più rilevanti e costose malattie sociali della nostra epoca, per la tendenza a determinare complicanze sia acute che croniche $[1,2]$ e per il progressivo spostamento dell'insorgenza verso età giovanili. Nel 2015 [3] l'International Diabetes Federation (IDF) stimava che gli adulti con diabete fossero 415 milioni; in base alle proiezioni, si ritiene che questo numero potrebbe aumentare fino a raggiungere i 642 milioni nel 2040. Nelle grandi città vivono oggi due terzi delle persone affette da diabete. Infatti secondo i dati dell'IDF, nel mondo sono 246 milioni (65\%), coloro che hanno ricevuto una diagnosi di diabete di Tipo 2 e abitano nei centri urbani, rispetto ai 136 milioni delle aree rurali. Questo divario è destinato a crescere: nel 2040 si stima che circa il $75 \%$ delle persone con diabete vivrà nelle città: 347 milioni rispetto ai 147 milioni che abiteranno fuori dai grandi centri abitati. Anche in Italia l'urban diabetes è un problema emergente di sanità pubblica, visto che nelle 14 città metropolitane risiede il 36\% della popolazione del Paese e circa 1,2 milioni di persone con diabete. Da qui la necessità di mettere in atto nelle aree urbane una strategia integrata, finalizzata a costruire un'idea di città come "promotore della salute", attraverso un approccio multilivello che comprenda iniziative di vario genere, sociali prima ancora che sanitarie. Interventi urbanistici, "laboratori" sugli stili di vita sani come Cities Changing Diabetes ${ }^{\circledR}$, già avviato a Città del Messico, Pechino, Houston, Copenaghen, Tianjin, Johannesburg, Vancouver e che vede coinvolta da quest'anno anche Roma, con un ruolo attivo del Ministero della Salute, dell'ANCI, di Roma Capitale e Città Metropolitana, dell'Health City Institute, dell'ISS, di ISTAT, della Fondazione CENSIS, di CORESEARCH, di MEDIPRAGMA, di Cittadinanzattiva, di tutte le Università di Roma, dell'Osservatorio Nazionale per la Salute, dell'Istituto per la Competitività nelle Regioni e di tutte le Società Scientifiche e Associazioni Pazienti in ambito diabetologico [4].

\section{Cities Changing Diabetes ${ }^{\circledR}$ il primo progetto mondiale di urban diabetes}

Cities Changing Diabetes ${ }^{\circledR}$ nasce nel 2014 in Danimarca ed è un programma di partnership promosso dallo University College of London (UK) e dallo Steno Diabetes Center (Danimarca) con il sostegno di Novo Nordisk, in collaborazione con partner nazionali che comprendono istituzioni, città metropolitane, comunità diabetologiche/sanitarie, amministrazioni locali, mondo accademico e terzo settore.
L'obiettivo del programma è quello di creare un movimento unitario in grado di stimolare, a livello internazionale e nazionale, i decisori politici a considerare come prioritario il tema dell'urban diabetes. Mettendo in luce il fenomeno con dati ed evidenze provenienti dalle città di tutto il mondo, il programma Cities Changing Diabetes ${ }^{\circledR}$ sottolinea la necessità di agire in considerazione del crescente numero di persone con diabete e del conseguente onere economico e sociale che tutto ciò comporta. Roma è stata inserita nel programma nel 2017, seconda città europea dopo Copenaghen, diventando per i prossimi anni oggetto di studi internazionali su urbanizzazione e diabete Tipo 2 e nello stesso tempo Città simbolo mondiale nella lotta a questa importante patologia.

In Italia l'Health City Institute, in collaborazione con Ministero della Salute e ANCI, ha promosso inoltre la realizzazione del Manifesto della Salute nelle Città, documento che delinea le azioni da intraprendere per studiare i determinati della salute nelle Città e migliorare la qualità di vita dei cittadini. Il Manifesto ha rappresentato la base per la redazione della proposta di iniziativa presentata dalla Delegazione Italiana del Comitato delle Regioni dell'Unione Europea alla Commissione NAT e al Parlamento Europeo e approvata in seduta plenaria l'11 maggio 2017.

Il progetto Cities Changing Diabetes ${ }^{\circledR}$ prevede tre momenti:

- il primo è rappresentato dalla mappatura dei fattori sociali e culturali. In ogni città viene promossa una ricerca qualitativa e quantitativa dalla quale emerge il livello di vulnerabilità.

- il secondo è quello della condivisione dei dati a livello internazionale, fra le varie città aderenti al programma, con l'obiettivo di identificare le similitudini ed i punti di contatto.

- la terza e ultima fase del programma è la parte dedicata all'azione, con lo sviluppo di uno specifico action plan e condivisione delle best practice.

L'Health City Institute, in collaborazione con la Fondazione CENSIS, l'ISTAT, CORESEARCH e MEDIPRAGMA ha elaborato i dati oggi disponibili a livello sociodemografico e clinico-epidemiologico e sulla percezione della salute nell'area di Roma Città Metropolitana, realizzando una prima mappatura dei dati quantitativi, pubblicati in un Atlas. La fase qualitativa, condotta tramite la Fondazione CENSIS, prevede lo studio delle vulnerabilità sociali dell'area metropolitana sul diabete Tipo 2, attraverso interviste strutturate sui cittadini e sulle persone con diabete di Roma.

\section{Il contesto socio-demografico di Roma}

Dal secondo dopoguerra l'area metropolitana di Roma ha conosciuto un'espansione demografica particolarmente rilevante. La popolazione raddoppia nella città metropolitana, 
passando da 2,1 milioni nel 1951 a 4,0 milioni nel 2011, e cresce di oltre il $70 \%$ nel solo comune capoluogo (da 1.650 .000 a 2.618 .000 nel 2011). A partire dai primi anni '70, l'espansione demografica della città metropolitana è contrassegnata, come nel resto del Paese, dall'invecchiamento della popolazione. L'indice di vecchiaia (rapporto percentuale tra ultrasessantacinquenni e bambini di 0-14 anni) passa da 35,1 nel 1971 a 144,2 nel 2011. Nello stesso periodo la quota di persone di almeno 75 anni passa da 2,9 a 9,7\%. Nel solo Comune la quota di ultrasettantacinquenni raggiunge il 10,7\% [5].

Secondo gli ultimi dati ISTAT (2016), nella città metropolitana di Roma risiedono 4,3 milioni di persone (2,9 nel Comune). Gli stranieri residenti sono oltre 500.000 (il $12,1 \%$ a fronte dell' $8,3 \%$ medio nazionale), di cui 365.000 in città (il 12,7\%). La speranza di vita alla nascita è di 80,4 anni per gli uomini e 84,7 per le donne (valori entrambi lievemente superiori alla media nazionale). A 65 anni la vita residua è di 22 anni per le donne e di 19 per gli uomini. I residenti con più di 65 anni sono quasi $900.000 \mathrm{e}$ 264.000 quelli con almeno ottant'anni (626.000e 193.000 nel Comune). Nel 2016 l'indice di vecchiaia ha raggiunto nel territorio comunale un valore pari a 162 , con marcate variabilità tra le diverse zone urbanistiche della città: in quelle più centrali-e comunque all'interno del grande raccordo anulare-si riscontrano valori dell'indicatore spesso superiori a 200.

I mutamenti demografici sono associati a profondi cambiamenti nella struttura della società, con differenze talora sostanziali tra contesti urbani e non. La dimensione media delle famiglie della città metropolitana si riduce: da 3,3 componenti nel 1971 a 2,2 nel 2011, non solo perché si fanno meno figli ma perché aumentano le persone sole (anziani, ma anche giovani). Il fenomeno è più accentuato nei sistemi urbani. A Roma nel 1971 poco più di una famiglia su 10 era unipersonale, nel 2011 lo è più di una famiglia su tre. In particolare, gli anziani (>64 anni) che vivono soli sono il $28,4 \%(29,4 \%$ nel territorio comunale, mentre la media italiana è $27,1 \%)$.

Una nota merita il capitolo dell'istruzione, partendo dal presupposto che il livello generale d'istruzione degli adulti è cresciuto ed è più elevato nei contesti urbani. A Roma nel 2011, per ogni persona con la licenza media ve n'erano più di tre con diploma o titolo universitario, mentre in Italia l'analogo rapporto è di uno a 1,6; questo livello era già stato raggiunto dal comune di Roma nel 1991. Per contro, la quota di persone tra i 15 e i 24 anni uscite dal sistema di formazione e istruzione è assai più contenuta $(9 \%)$ rispetto alla media regionale $(11,8 \%)$ e italiana $(15,5 \%)$. In ambito cittadino il livello d' istruzione è sensibilmente più elevato nei quartieri centrali.

Altro indice che si può correlare alla salute è quello della mobilità. In base ai dati censuari, ogni giorno il 68,3\% della popolazione cittadina si sposta per motivi di studio (in circa due terzi dei casi) o di lavoro. Si tratta di circa 1.339.000 persone che in larghissima parte rimangono all'interno del comune di Roma (il 95,8\%). Per circa un terzo dei residenti che rimangono all'interno del comune lo spostamento è inferiore ai 15 minuti (ma solo per il 23\% di chi si sposta per lavoro). D'altro canto per più di una persona su cinque lo spostamento è superiore a 45 minuti. È evidente lo svantaggio rispetto alla media italiana con il $55 \%$ di spostamenti al di sotto dei 15 minuti e solo il 10,7\% sopra i 45 minuti. Per gli spostamenti quotidiani per lavoro e studio il 59\% delle persone utilizza principalmente un mezzo privato veloce (automobile, moto, motorino, ecc.). Circa il $15 \%$ lo fa a piedi o in bicicletta (cosiddetta mobilità lenta) e il rimanente $26 \%$ utilizza un mezzo pubblico. Il modello di mobilità urbana si discosta da quello prevalente a livello italiano per un maggior uso del mezzo pubblico a scapito sia della mobilità lenta sia del mezzo privato.

\section{Lo stato di salute dei servizi alla persona nell'area metropolitana di Roma}

All'interno dell'indagine quantitativa su Roma Cities Changing Diabetes ${ }^{\circledR}$, Medi-Pragma ha svolto un'indagine con l'obiettivo di analizzare la percezione dei cittadini residenti in diverse aree urbane in merito alla presenza di servizi rivolti alla cittadinanza. Dall'inchiesta emerge che il tema della salute è considerato il più importante da tutti gli intervistati, con una maggiore attribuzione d'importanza nell'hinterland romano rispetto alle altre province del Lazio. A seguire, un'occupazione garantita e la sicurezza della città risultano essere caratteristiche fondamentali per la vivibilità della stessa. Un giudizio intermedio viene attribuito alla tutela dell'infanzia. La tutela delle fasce più deboli, i trasporti e il traffico sono caratteristiche importanti, ma considerate in secondo piano rispetto alla salute e la sicurezza.

I servizi sanitari disponibili sul territorio regionale sono giudicati poco soddisfacenti da parte dei cittadini in base alla loro esperienza, soprattutto nelle aree extra-urbane della città di Roma. Le aree verdi, le piste ciclabili e le zone pedonali sono poco considerate come strutture funzionali allo stato di salute e al benessere della persona, soprattutto nelle zone del Centro di Roma, e i cittadini ne richiedono una maggiore efficienza, laddove presenti. Roma sembra non essere un luogo particolarmente attento alle categorie a rischio e ai disabili. Le strutture deputate al benessere degli anziani e delle persone disabili sono estremamente carenti. Nonostante lo stato dell' arte, le fasce più giovani della popolazione che vivono fuori dal centro di Roma, nelle zone limitrofe o nelle province, considerano mediamente il loro ambiente favorevole alla salute e alla sicurezza e adatto a soddisfare l'ambizione lavorativa. 
Fig. 1 La prevalenza del diabete nei distretti sanitari dell'area metropolitana di Roma. Fonte: Programma Regionale Valutazione degli Esiti degli Interventi Sanitari 2016

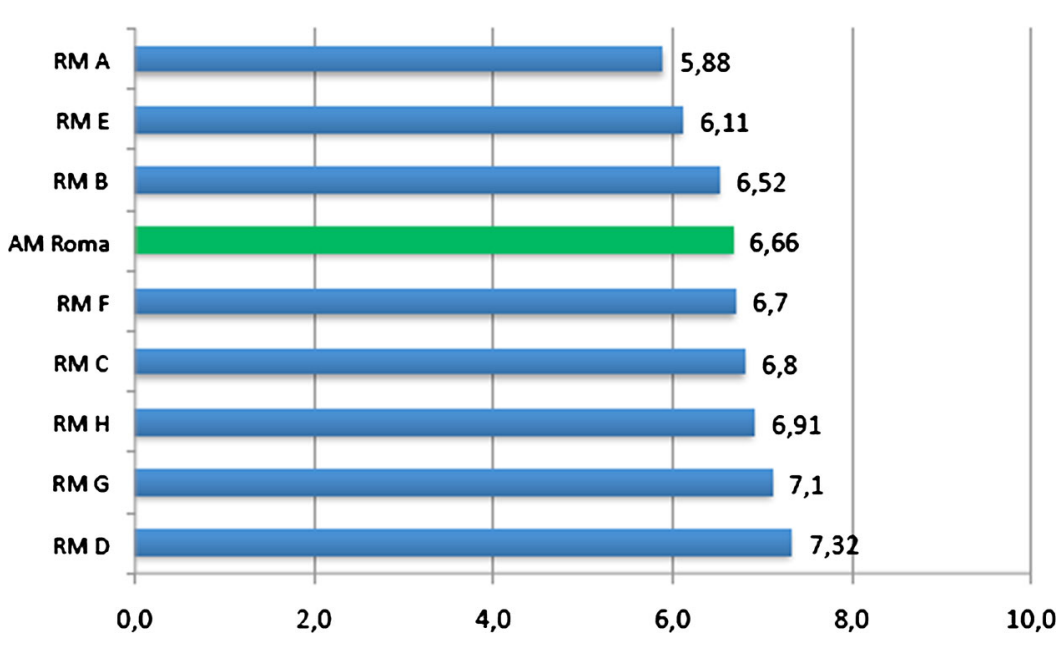

\section{La dimensione epidemiologica del diabete Tipo 2 e dell'obesità nell'area di Roma città metropolitana}

Sulla base dei dati ISTAT, nel 2015 la prevalenza del diabete nel Lazio era del 6,6\%. Rispetto al 2000, la prevalenza è cresciuta dal 5,0 al 6,5\% fra gli uomini e dal $4,2 \%$ al $6,8 \%$ nelle donne. Il Lazio è fra le regioni a più alta prevalenza di diabete, preceduto solo da Calabria e Campania. A fronte di una prevalenza media nazionale del 5,4\%, nel Lazio la prevalenza sale al 6,6\%. Applicando tale prevalenza alla popolazione residente nel Lazio nel 2015 (5.892.425 cittadini), si stima che 388.900 persone siano affette da diabete noto. Considerando che nella città di Roma risiedono 2.872.021 persone e nell' area metropolitana di Roma 4.340.474, si può stimare che nella città di Roma siano presenti circa 189.500 persone con diabete e nell' area metropolitana circa 286.500. In altre parole, la metà delle persone con diabete residenti nel Lazio vive a Roma e il 75\% vive nell' area metropolitana di Roma.

Sulla base delle stime fatte nell'ambito del Programma Regionale Valutazione degli Esiti degli Interventi Sanitari 2016 [6], all'interno dell'area metropolitana di Roma è presente una marcata variabilità nella prevalenza di diabete noto, che oscilla fra il 5,88 al 7,32\% nei diversi distretti sanitari (Fig. 1).

Come già discusso, uno dei fattori più importanti alla base della crescita della prevalenza di diabete è rappresentato dall'invecchiamento della popolazione. Nella città di Roma, il numero di ultrasessantacinquenni è cresciuto di 136.000 unità negli ultimi 13 anni, raggiungendo la quota di 631.000 residenti nel 2015.

L'altro fattore principale alla base dell' aumento della prevalenza del diabete è rappresentato dall'obesità. Nel Lazio, un residente su 10 in età adulta è affetto da obesità. Analogamente alla prevalenza del diabete, anche quella dell'obesità è cresciuta negli anni più recenti, passando dall' $8,2 \%$ nel 2000 al 9,9\% nel 2013. Oltre alle errate abitudini alimentari, la sedentarietà rappresenta uno dei determinanti dell'eccesso ponderale. I dati ISTAT mostrano come nel Lazio oltre il
$40 \%$ della popolazione sia totalmente sedentaria, mentre solo un quinto dei cittadini svolge attività fisica con regolarità. In tutte le fasce di età, la percentuale di persone sedentarie risulta strettamente correlata alla scolarità, considerata come indicatore di stato socioeconomico [7].

Il diabete ha un importante impatto sulla aspettativa di vita. In Italia si stima che ogni anno oltre 20.000 decessi siano attribuibili direttamente al diabete, mentre in ulteriori 75.000 decessi il diabete è presente come concausa. I dati standardizzati $(\times 10.000)$ di mortalità per diabete nel Lazio mostrano un trend di riduzione significativo, sia negli uomini che nelle donne. Mentre nella prima metà degli anni 2000 la mortalità nel Lazio era marcatamente più elevata rispetto alla media nazionale, negli ultimi anni il divario si è notevolmente ridotto [8].

\section{Conclusioni}

La condivisione dei risultati è fondamentale al fine di proporre azioni che possano impattare la salute di molti cittadini nella città di Roma. L'obiettivo del programma Cities Changing Diabetes ${ }^{\circledR}$ è di condividere esperienze di paesi e città diverse per consentire uno scambio di esperienze positive. Altrettanto importante appare l'identificazione e l'implementazione su larga scala di soluzioni per contrastare l'espansione del diabete di Tipo 2 e dell'obesità nell'area metropolitana di Roma: è questo l'obiettivo più importante che si prefigge il programma Cities Changing Diabetes ${ }^{\circledR}$. A questo fine, è necessario studiare attentamente le vulnerabilità come elemento per definire le azioni future. Vulnerabilità che si compone di almeno tre elementi, quali la suscettibilità individuale, la condizione sociale e i determinanti culturali. Mettendo in relazione la vulnerabilità con la regola dei mezzi emerge che la condizione in cui viviamo è di fondamentale importanza nel rischio di sviluppare il diabete di Tipo 2 e nel determinare i risultati a lungo termine della malattia. 
L'inurbamento e la configurazione attuale delle città offrono per la salute pubblica e individuale tanti rischi, ma anche opportunità da sfruttare con un'amministrazione cosciente e oculata; occorre pertanto identificare strategie di azione per rendere consapevoli governi, regioni, città e cittadini dell'importanza della promozione della salute nei contesti urbani, immaginando un nuovo modello di welfare urbano. Questa trasformazione, inevitabilmente, richiede una comprensione e un' analisi attenta dello scenario futuro. Le Società Scientifiche, in particolare quelle che operano in ambito endocrino-metabolico, avranno un ruolo fondamentale nella conduzione e nell'interpretazione della suddetta analisi al fine di elaborare le corrette politiche per un miglioramento della salute dei cittadini.

Conflitto di interesse Gli autori A. Lenzi, F. Dotta, S. Frontoni, R. Crialesi, A. Nicolucci e K. Vaccaro dichiarano di non avere conflitti di interesse.

Consenso informato Lo studio presentato in questo articolo non ha richiesto sperimentazione umana.

Studi su animali Il contenuto del presente articolo non riguarda studi effettuati su animali.

\section{Bibliografia}

1. Annali AMD VIII Edizione. http://aemmedi.it/annali/ (Accessed 6 July 2017)

2. Penno G, Solini A, Bonora E et al (2013) Gender differences in cardiovascular disease risk factors, treatments and complications in patients with type 2 diabetes: the RIACE Italian multicentre study. J Intern Med 274:176-191

3. International Diabetes Federation Atlas, 7th edn. http://www. diabetesatlas.org/ (Accessed 6 July 2017)

4. Roma Cities Changing Diabetes: Diabete Tipo 2 e Obesità nell'area di Roma Città Metropolitana Factsheets Atlas 2017. http://healthcitythinktank.org/ATLAS-Factsheets-Aprile-2017.pdf (Accessed 6 July 2017)

5. ISTAT (2011) Censimento della popolazione e delle abitazioni. https://www.istat.it/it/censimento-popolazione/censimentopopolazione-2001 (Accessed 7 July 2017)

6. Programma Regionale Valutazione degli Esiti degli Interventi Sanitari 2016. http://95.110.213.190/prevale2016/territoriale/ territoriale.php (Accessed 6 July 2017)

7. ISTAT (2016) Indagine multiscopo aspetti della vita quotidiana. https://www.istat.it/it/archivio/129916 (Accessed 7 July 2017)

8. ISTAT (2017) Indagine sui decessi e le cause di morte. https://www.istat.it/it/archivio/4216 (Accessed 7 July 2017) 